\title{
WCVaR-Based Risk Coordination Model for Multi-Product Supply
}

\author{
Min Jiang, Rui Shen, Zhiqing Meng \\ College of Economics and Management, Zhejiang University of Technology, Hangzhou, China \\ Email: jiangmin9@126.com, shenrui126@126.com, mengzhiqing@zjute.edu.cn
}

How to cite this paper: Jiang, M., Shen, R. and Meng, Z.Q. (2017) WCVaR-Based Risk Coordination Model for Multi-Product Supply. Open Journal of Business and Management, 5, 641-652. https://doi.org/10.4236/ojbm.2017.54054

Received: August 6, 2017

Accepted: September 15, 2017

Published: September 18, 2017

Copyright @ 2017 by authors and Scientific Research Publishing Inc. This work is licensed under the Creative Commons Attribution International License (CC BY 4.0).

http://creativecommons.org/licenses/by/4.0/

\section{(c) (i) Open Access}

\begin{abstract}
The paper develops a multi-product supply chain model where supplyproduction-sale integration is considered and the worst-case conditional value at risk (WCVaR) model is applied as the risk measure, and also provides a coordination strategy to minimize the supply chain risk. First, by analyzing the source demand of market in the supply chain, three WCVaR models consisting of three tiers-the supplier, the manufacturer and the retailer in the supply chain are proposed to measure the market risk. Then, a risk coordination model is proposed to cover the whole supply chain including producing, order, inventory and sales. Finally, the numerical results show the efficiency of the model in mitigating risks. And we make a summary of supply chain risk management strategies.
\end{abstract}

\section{Keywords}

WCVaR Model, Supply Chain, Supply-Production-Sale Integration, Multi Product

\section{Introduction}

The early research on integrative supply chain focused on the condition of certain demand with problems solved by integer programming models. In recent years scholars have begun to study the condition of random demand which is more close to reality [1]-[6].

Since Rockafellar and Uryasev [7] [8] proposed the equivalent model of CVaR, it has been widely used in supply chain risk management [9] [10] [11], and etc. Gotoh et al. [9] proposed analytical solutions and linear programming (LP) formulation of the minimization of the CVaR measures defined with two different loss functions and demonstrated the efficiency of the LP solutions by numerical examples. Zhang et al. [10] presented some convex stochastic programming 
models for single- and multi-period inventory control problems to minimize the expected losses subject to risk aversion constraints expressed through $\mathrm{VaR}$ and $\mathrm{CVaR}$ as risk measures, and proposed a sample average approximation method. Zhou et al. [11] proposed an optimal order model for multi-product problems, which is formulated as a linear programming problem subject to CVaR constraints and offers an appropriate analytical tool for decision-makers with different risk preferences. On this basis, we would like to have the concept of WCVaR, which was introduced by Zhu et al. [12] for the situation where the probability distribution are partially known; it is proved that the WCVaR inherits the same properties of the $\mathrm{CVaR}$ such as the coherency and the convexity, which makes risk analysis available and makes it an effective tool for supply chain risk measurement.

In this paper, two types of risks are included to represent the total risk in supply chain. And the coordination risk of supply chain is expressed with the equilibrium between the minimization of market risk and of operation risk. In addition, as mentioned above, CVaR/WCVaR has several fine mathematical properties [7] [12]. So the paper is organized as follows: Section 2 studies three market risk models and proposes the coordination model with the robust optimization models via WCVaR; in Sections 3, the numerical examples are presented to test the models and the theoretical analysis; Section 4 draws some conclusions.

\section{Supply Chain Risk Modeling}

\subsection{Description of the Problem}

The supply chain we consider includes retailers, manufacturer, suppliers and customers. The process is divided into three phases, and the risk models are proposed via WCVaR: (1) retailer's order-sales model; (2) manufacturer's order-production-wholesale model; (3) supplier's stock-supply model in Figure 1. Then we have the following assumptions:

Assumption 1: All enterprises make decisions based on the customers' demand, and the demands for all goods and raw materials are independent of each other.

Assumption 2: If the goods are not sold out at the end of the period, retailers will sell the rest at a discount price. So do manufacturers.

Assumption 3: In this paper, two kinds of risk are considered: one is called operation risk and the other is called market risk. The former is aroused mainly due to the uncertainty between the upstream and downstream enterprises; while the latter is mainly due to the uncertainty between the first three in the supply chain and the customer. The total risk of supply chain is considered as the sum of these two risks.

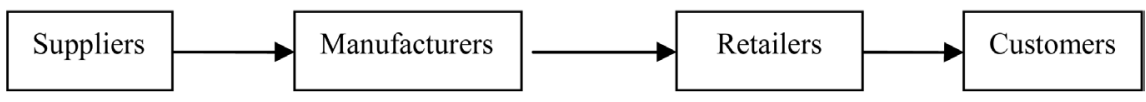

Figure 1. Supply chain model. 


\subsection{Modeling}

Based on the above assumptions, we divide the whole process of supply chain into three phases to build the following corresponding WCVaR models:

\section{1) Retailer's order-sales model}

This is the phase where most of market risks in supply chain is found. The retailer orders from the manufacturer based on customers' demand. Variables are as follows: let the customer's demand vector for all products is represented as $\xi=\left(\xi_{1}, \cdots, \xi_{n}, \cdots, \xi_{N}\right)^{\mathrm{T}}$; let $x_{n}$ denote the retailer's order quantity for product $n$, and the retailer's order quantity vector for all products is represented as $\boldsymbol{x}=\left(x_{1}, \cdots, x_{n}, \cdots, x_{N}\right)^{\mathrm{T}}$; let $X$ denote the order set from the retailer; suppose that the loss function in the first phase is $L_{1}\left(x, \xi^{i}\right)$, and the random variable $\xi^{i}$ yields a distribution density function $p_{1}^{i}(t)(i=1,2, \cdots, I)$. Therefore, we define the customer's joint distribution clusters of the demand as:

$$
\mathrm{P}_{1}=\left\{\sum_{i=1}^{I} \lambda_{i}^{1} p_{1}^{i}(t) \mid \sum_{i=1}^{I} \lambda_{i}^{1}=1, \lambda_{i}^{1} \geq 0, i=1,2, \cdots, I\right\}
$$

Let $\Lambda_{1}=\left\{\lambda_{1}=\left(\lambda_{1}^{1}, \lambda_{2}^{1}, \cdots, \lambda_{I}^{1}\right) \mid \sum_{i=1}^{I} \lambda_{i}^{1}=1, \lambda_{i}^{1} \geq 0, i=1,2, \cdots, I\right\}$, we define

WCVaR as follows:

$$
\operatorname{WCVaR}_{\beta_{1}}(x)=\max _{\lambda \in \Lambda_{1}} \sum_{i=1}^{I} \lambda_{i} F_{1 i}\left(x, \alpha_{1}\right)
$$

where a CVaR loss function $F_{1 i}\left(x, \alpha_{1}\right)=\alpha_{1}+\frac{1}{1-\beta_{1}} \int_{t \in R^{m}}\left[L_{1}(x, t)-\alpha_{1}\right]^{+} p_{1}^{i}(t) \mathrm{d} t$.

The retailer's optimal order quantity can be solved from:

$$
\begin{array}{ll}
\min & \operatorname{WCVaR}_{\beta_{1}}(\boldsymbol{x})=\max _{\lambda_{1} \in \Lambda_{1}} \sum_{i=1}^{I} \lambda_{i}^{1} F_{1 i}\left(\boldsymbol{x}, \alpha_{1}\right) \\
\text { s.t. } & \boldsymbol{x} \in X
\end{array}
$$

which is equivalent to

$$
\begin{array}{ll}
\min & \chi_{1} \\
\text { s.t. } & \chi_{1} \geq F_{1 i}\left(x, \alpha_{1}\right), i=1,2, \cdots, I, \\
& x \in X, \alpha_{1}, \chi_{1} \in R^{1} .
\end{array}
$$

Suppose that $c_{n}$ denotes retailer's order cost per unit for product $n ; r_{n}$ denotes retailer's salvage value per unit for product $n$, where $r_{n}<c_{n} ; a_{n}$ denotes retailer's inventory cost per unit for product $n ; s_{n}$ denotes retailer's selling price per unit for product $n$. For retailer, the total loss of all products includes inventory loss and discount loss when there is oversupply, as well as the opportunity loss when the product is in short supply. So in this phase the loss function can be expressed as follows:

$$
L_{1}(\boldsymbol{x}, \boldsymbol{\xi})=\sum_{n=1}^{N}\left\{\left(a_{n}+c_{n}-r_{n}\right)\left[x_{n}-\xi_{n}\right]^{+}+\left(s_{n}-c_{n}\right)\left[\xi_{n}-x_{n}\right]^{+}\right\}
$$

Let $\xi_{k}$ denotes random vector $\boldsymbol{\xi}$ at time $k$, where $k=1, \cdots, K$, and $p_{1 k}^{i}$ denotes the probability distribution of samples. So the approximate expression 
of $F_{1 i}\left(x, \alpha_{1}\right)$ is represented as follows $i=1, \cdots, I$ :

$$
F_{1 i}\left(\boldsymbol{x}, \alpha_{1}\right) \approx \tilde{F}_{1 i}\left(\boldsymbol{x}, \lambda_{1}, \alpha_{1}\right)=\alpha_{1}+\frac{1}{1-\beta_{1}} \sum_{k=1}^{K}\left(\left[L_{1}\left(\boldsymbol{x}, \boldsymbol{\xi}_{k}\right)-\alpha_{1}\right]^{+} p_{1 k}^{i}\right) .
$$

Let $\mu_{1, k}=L_{1}\left(x, \xi_{k}\right)-\alpha_{1}, \quad v_{1, k}=x_{n}-\xi_{n, k}$, and $\omega_{1, k}=\xi_{n, k}-x_{n}, \quad k=1, \cdots, K$.

Suppose that $\mu_{1}=\left(\mu_{1,1}, \mu_{1,2}, \cdots, \mu_{1, K}\right), \quad v_{1}=\left(v_{1,1}, v_{1,2}, \cdots, v_{1, K}\right)$,

$\boldsymbol{\omega}_{1}=\left(\omega_{1,1}, \omega_{1,2}, \cdots, \omega_{1, K}\right)$. At a certain confidence level of $\beta_{1}$, we have the following linear programming problem:

$$
\begin{gathered}
\text { (WCVaR 1) } \begin{array}{c}
\min _{\left(x, \alpha_{1}, \mu_{1}, \nu_{1}, \omega_{1}, \chi_{1}\right)} \\
\text { s.t. } \mu_{1, k} \geq \sum_{n=1}^{N}\left\{\left(a_{n}+c_{n}-r_{n}\right) v_{1, k}+\left(s_{n}-c_{n}\right) \omega_{1, k}\right\}-\alpha_{1}, \\
\mu_{1, k} \geq 0, \quad v_{1, k} \geq x_{n}-\xi_{n, k}, \quad v_{1, k} \geq 0, \\
\quad \omega_{1, k} \geq \xi_{n, k}-x_{n}, \quad \omega_{1, k} \geq 0, \\
\sum_{n=1}^{N} c_{n} x_{n} \leq \Phi_{1}, \quad \alpha_{1}, \chi_{1} \in R^{1}, \quad A_{n}^{1} \leq x_{n} \leq A_{n}^{2}, \\
\chi_{1} \geq \alpha_{1}+\frac{1}{1-\beta_{1}} \sum_{k=1}^{K} \mu_{1, k} p_{1 k}^{i}, \quad i=1,2, \cdots, I, \\
n=1, \cdots, N, \quad k=1, \cdots, K .
\end{array}
\end{gathered}
$$

The linear programming problem (WCVaR 1) minimizes the worst-case conditional value at risk for the decision-maker who takes inventory loss and opportunity loss of all products in the supply chain into consideration. In addition, $\Phi_{1}$ denotes the financial budget for the retailer's orders. Finally $A_{n}^{1}$ and $A_{n}^{2}$ denote the least and the most order quantity respectively. Then we can get the approximate (WCVaR) and the corresponding optimal order $x^{*}$ for retailer by solving problem (WCVaR 1).

\section{2) Manufacturer's order-production-wholesale model}

In this phase, the manufacturer orders a quantity of raw materials from the supplier for production. The variables are as follows: let random variable $\xi=\left(\xi_{1}, \cdots, \xi_{n}, \cdots, \xi_{N}\right)^{\mathrm{T}}$; let $x_{n}$ denote the manufacturer's production quantity for product $n$, and the manufacturer's production quantity vector for all products can be represented as $\boldsymbol{y}=\left(y_{1}, \cdots, y_{n}, \cdots, y_{N}\right)^{\mathrm{T}}$; let $Y$ denote production set for the manufacturer; suppose that the loss function in the second phase can be represented as $L_{2}\left(y, \zeta^{i}\right)$, and the random variable $\zeta^{i}$ yields the distribution density function $p_{2}^{i}(t)$. We define the manufacturer's joint distribution clusters of the demand as:

$$
\mathrm{P}_{2}=\left\{\sum_{i=1}^{I} \lambda_{i}^{2} p_{2}^{i}(t) \mid \sum_{i=1}^{I} \lambda_{i}^{2}=1, \lambda_{i}^{2} \geq 0, i=1,2, \cdots, I\right\} .
$$

Let $\Lambda_{2}=\left\{\lambda_{2}=\left(\lambda_{1}^{2}, \lambda_{2}^{2}, \cdots, \lambda_{I}^{2}\right) \mid \sum_{i=1}^{I} \lambda_{i}^{2}=1, \lambda_{i}^{2} \geq 0, i=1,2, \cdots, I\right\}$, we define

WCVaR as follows: 


$$
\operatorname{WCVaR}_{\beta_{2}}(\boldsymbol{y})=\max _{\lambda_{2} \in \Lambda_{2}} \sum_{i=1}^{I} \lambda_{i}^{2} F_{2 i}\left(\boldsymbol{y}, \alpha_{2}\right),
$$

where $F_{2 i}\left(y, \alpha_{2}\right)=\alpha_{2}+\frac{1}{1-\beta_{2}} \int_{t \in R^{m}}\left[L_{2}(y, t)-\alpha_{2}\right]^{+} p_{2}^{i}(t) \mathrm{d} t$. The manufacturer's optimal production quantity can be solved from:

$$
\begin{array}{ll}
\min & \operatorname{WCVaR}_{\beta_{2}}(\boldsymbol{y})=\max _{\lambda_{2} \in \Lambda_{2}} \sum_{i=1}^{I} \lambda_{i}^{2} F_{2 i}\left(\boldsymbol{y}, \alpha_{2}\right) \\
\text { s.t. } & \boldsymbol{y} \in Y
\end{array}
$$

which is equivalent to

$$
\begin{array}{ll}
\min & \chi_{2} \\
\text { s.t. } & \chi_{2} \geq F_{2 i}\left(y, \alpha_{2}\right), i=1,2, \cdots, I, \\
& y \in Y, \alpha_{2}, \chi_{2} \in R^{1} .
\end{array}
$$

According to assumption, the retailer's demand correlates with the source demand-consumers' demand, a fluctuation factor. So we have $\zeta=\varphi_{1}(\xi)$. For simplicity, let $\boldsymbol{\zeta}=\boldsymbol{\xi}+\boldsymbol{\theta}$, where $\boldsymbol{\theta}$ denotes the fluctuation factor, and for product $n, \theta_{n}(n=1, \cdots, N)$ is a random variable which yields the normal distribution $N\left(0, \delta^{2}\right)$, which are independent of the source demand. In addition, we suppose that $l_{n}$ denotes manufacturer's production cost per unit for product $n ; g_{n}$ denotes manufacturer's salvage value per unit for product $n$, where $g_{n}<l_{n} ; b_{n}$ denotes manufacturer's inventory cost per unit for product $n ; c_{n}$ denotes retailer's selling price per unit for product $n ; w_{m}$ denotes manufacturer's order cost per unit for material $m$ (given); $A_{m^{*} n}$ denotes the required quantity of material $m$ for per unit of product $n$, so for all products, the total demand for material $m$ is $\sum_{n=1}^{N} A_{m^{*}} y_{n} ; v_{n}$ denotes the processing cost per unit for product $n$ (given); so we have $l_{n}=v_{n}+\sum_{m=1}^{M} w_{m} A_{m^{*} n}$. For the manufacturer, the total loss of all products includes inventory loss and discount loss in case of oversupply, as well as the opportunity loss in case of short supply. So in this phase the loss function can be expressed as follows:

$$
L_{2}(\boldsymbol{y}, \boldsymbol{\zeta})=\sum_{n=1}^{N}\left\{\left(b_{n}+l_{n}-g_{n}\right)\left[y_{n}-\boldsymbol{\zeta}_{n}\right]^{+}+\left(c_{n}-l_{n}\right)\left[\boldsymbol{\zeta}_{n}-y_{n}\right]^{+}\right\} .
$$

Let $\zeta_{k}$ denote random vector $\zeta$ at time $k$, where $k=1, \cdots, K$, and $p_{2 k}^{i}$ denote the probability distribution of samples. So the approximate expression of $F_{2}\left(y, \alpha_{2}\right)$ is represented as follow:

$$
\tilde{F}_{2}\left(y, \lambda_{2}, \alpha_{2}\right)=\alpha_{2}+\frac{1}{1-\beta_{2}} \sum_{k=1}^{K}\left(\left[L_{2}\left(y, \zeta_{k}\right)-\alpha_{2}\right]^{+} \sum_{i=1}^{I} \lambda_{i}^{2} p_{2 k}^{i}\right) .
$$

Let $\mu_{2, k}=L_{2}\left(y, \zeta_{k}\right)-\alpha_{2}, \quad v_{2, k}=y_{n}-\zeta_{n, k}$, and $\omega_{2, k}=\zeta_{n, k}-y_{n}, \quad k=1, \cdots, K$. According to the assumption, $\zeta_{n, k}=\xi_{n, k}+\theta_{n, k}$, where $k=1, \cdots, K$. Suppose $\boldsymbol{\mu}_{2}=\left(\mu_{2,1}, \mu_{2,2}, \cdots, \mu_{2, K}\right), \quad \boldsymbol{v}_{2}=\left(v_{2,1}, v_{2,2}, \cdots, v_{2, K}\right), \boldsymbol{\omega}_{2}=\left(\omega_{2,1}, \omega_{2,2}, \cdots, \omega_{2, K}\right)$. At a certain confidence level of $\beta_{2}$, we have the following linear programming 
problem:

(WCVaR 2)

$$
\begin{gathered}
\min _{\left(y, \alpha_{2}, \mu_{2}, v_{2}, \omega_{2}, \chi_{2}\right)} \chi_{2} \\
\text { s.t. } \mu_{2, k} \geq \sum_{n=1}^{N}\left\{\left(b_{n}+l_{n}-g_{n}\right) v_{2, k}+\left(c_{n}-l_{n}\right) \omega_{2, k}\right\}-\alpha_{2}, \\
\mu_{2, k} \geq 0, \quad v_{2, k} \geq y_{n}-\left(\xi_{n, k}+\theta_{n, k}\right), \quad v_{2, k} \geq 0, \\
\omega_{2, k} \geq\left(\xi_{n, k}+\theta_{n, k}\right)-y_{n}, \quad \omega_{2, k} \geq 0 \\
\sum_{n=1}^{N} l_{n} y_{n} \leq \Phi_{2}, \quad \alpha_{2}, \chi_{2} \in R^{1}, \\
\quad l_{n}=v_{n}+\sum_{m=1}^{M} w_{m} A_{m \times n}, \\
\chi_{2} \geq \alpha_{2}+\frac{1}{1-\beta_{2}} \sum_{k=1}^{K} \mu_{2, k} p_{2 k}^{i}, \quad i=1, \cdots, I, \\
A_{n}^{3} \leq y_{n} \leq A_{n}^{4}, \quad n=1, \cdots, N, \quad k=1, \cdots, K .
\end{gathered}
$$

The linear programming problem (WCVaR 2) minimizes the worst-case conditional value at risk-the objective of the decision-maker who takes inventory loss and opportunity loss of all products in the supply chain into consideration. In addition, $\Phi_{2}$ denotes the financial budget of the manufacturer for producing all products. Finally $A_{n}^{3}$ and $A_{n}^{4}$ denote the least and the most production quantity respectively. Then we can get the approximate WCVaR and the optimal production $\boldsymbol{y}^{*}$ (the corresponding quantity for raw material $m$ is $A_{m^{*} n} y^{*}$ for the manufacturer.

\section{3) Supplier's stock-supply model}

In this phase the supplier offers raw materials to the manufacturer. The variables are as follows: let random variable $\gamma=\left(\gamma_{1}, \cdots, \gamma_{m}, \cdots, \gamma_{M}\right)^{\mathrm{T}}$; let $z_{m}$ denote the supplier's inventory quantity for raw material $m$, and the supplier's inventory quantity vector for all raw materials can be represented as $\mathbf{Z}=\left(z_{1}, \cdots, z_{m}, \cdots, z_{M}\right)^{\mathrm{T}}$; let $Z$ denotes inventory set for the supplier; suppose that the loss function in the third phase can be represented as $L_{3}\left(z, \gamma^{i}\right)$, and the random variable $\gamma^{i}$ yields the distribution density function $p_{3}^{i}(t)$. We define the supplier's joint distribution clusters of the demand:

$$
\mathrm{P}_{3}=\left\{\sum_{i=1}^{I} \lambda_{i}^{3} p_{3}^{i}(t) \mid \sum_{i=1}^{I} \lambda_{i}^{3}=1, \lambda_{i}^{3} \geq 0, i=1,2, \cdots, I\right\} .
$$

Let $\Lambda_{3}=\left\{\lambda_{3}=\left(\lambda_{1}^{3}, \lambda_{2}^{3}, \cdots, \lambda_{I}^{3}\right) \mid \sum_{i=1}^{I} \lambda_{i}^{3}=1, \lambda_{i}^{3} \geq 0, i=1,2, \cdots, I\right\}$, we define

WCVaR as follows:

$$
\operatorname{WCVaR}_{\beta_{3}}(\boldsymbol{y})=\max _{\lambda_{3} \in \Lambda_{3}} \sum_{i=1}^{I} \lambda_{i}^{3} F_{3 i}\left(\mathbf{z}, \alpha_{3}\right),
$$

where $F_{3 i}\left(\mathbf{z}, \alpha_{3}\right)=\alpha_{3}+\frac{1}{1-\beta_{3}} \int_{t \in R^{m}}\left[L_{3}(\mathbf{z}, t)-\alpha_{3}\right]^{+} p_{3}^{i}(t) \mathrm{d} t$. The supplier's optimal stock quantity can be formulated by: 


$$
\begin{array}{ll}
\min & \operatorname{WCVaR}_{\beta_{3}}(\mathbf{z})=\max _{\lambda_{3} \in \Lambda_{3}} \sum_{i=1}^{I} \lambda_{i}^{3} F_{3 i}\left(\mathbf{z}, \alpha_{3}\right) \\
\text { s.t. } & \mathbf{z} \in X
\end{array}
$$

which is equivalent to

$$
\begin{array}{ll}
\min & \chi_{3} \\
\text { s.t. } & \chi_{3} \geq F_{3 i}\left(\mathbf{z}, \alpha_{3}\right), i=1,2, \cdots, I, \\
& \mathbf{z} \in Y, \alpha_{3}, \chi_{3} \in R^{1} .
\end{array}
$$

According to assumption, the manufacturer's demand correlates with the source demand-customers' demand, a fluctuation factor. So we have $\gamma=\varphi_{2}(\xi)$. For simplicity, let $\gamma=A_{m^{*} n}(\xi+\varepsilon) \quad$ (where $\sum_{n=1}^{N} A_{m^{*} n} \xi$ denotes the total stock quantity of raw material $m, \varepsilon$ denotes the fluctuation factor, and for product $n, \varepsilon_{n}$ is a random variable which yields the normal distribution $N\left(0, \sigma^{2}\right), n=1, \cdots, N$. In addition, they are independent of customer's demand). In addition, we suppose that $d_{m}$ denotes supplier's inventory cost per unit for raw material $m ; w_{m}$ denotes supplier's selling price per unit for raw material $m$ (given). So in this phase the loss function can be expressed as follows:

$$
L_{3}(\mathbf{z}, \boldsymbol{\gamma})=\sum_{m=1}^{M}\left\{d_{m}\left[z_{m}-\gamma_{m}\right]^{+}+w_{m}\left[\gamma_{m}-z_{m}\right]^{+}\right\} \text {. }
$$

Let $\gamma_{k}$ denote random vector $\gamma$ at time $k$, where $k=1, \cdots, K$, and $p_{3 k}^{i}$ denote the probability distribution of samples. So the approximate expression of $F_{3}\left(\mathbf{z}, \alpha_{3}\right)$ is represented as follows:

$$
\tilde{F}_{3}\left(\mathbf{z}, \lambda_{3}, \alpha_{3}\right)=\alpha_{3}+\frac{1}{1-\beta_{3}} \sum_{k=1}^{K}\left(\left[L_{3}\left(\mathbf{z}, \boldsymbol{\gamma}_{k}\right)-\alpha_{3}\right]^{+} \sum_{i=1}^{I} \lambda_{i}^{3} p_{3 k}^{i}\right) \text {. }
$$

Let $\mu_{3, k}=L_{3}\left(\mathbf{z}, \gamma_{k}\right)-\alpha_{3}, \quad v_{3, k}=z_{m}-\gamma_{m, k}$, and $\omega_{3, k}=\gamma_{m, k}-z_{m}, \quad k=1, \cdots, K$. According to the assumption, $\zeta_{n, k}=\xi_{n, k}+\theta_{n, k}$, where $k=1, \cdots, K$. Suppose that $\mu_{3}=\left(\mu_{3,1}, \mu_{3,2}, \cdots, \mu_{3, K}\right), \quad v_{3}=\left(v_{3,1}, v_{3,2}, \cdots, v_{3, K}\right), \omega_{3}=\left(\omega_{3,1}, \omega_{3,2}, \cdots, \omega_{3, K}\right)$. According to the assumption we have $\gamma_{m, k}=\sum_{n=1}^{N}\left[A_{m^{*} n}\left(\xi_{n, k}+\varepsilon_{n, k}\right)\right]$. At a certain confidence level of $\beta_{3}$, we have the following linear programming problem:

$$
\begin{gathered}
\text { (WCVaR 3) } \min _{\left(z, \alpha_{3}, \mu_{3}, \nu_{3}, \omega_{3}, \chi_{3}\right)} \chi_{3} \\
\text { s.t. } \mu_{3, k} \geq \sum_{m=1}^{M}\left\{d_{m} v_{3, k}+w_{m} \omega_{3, k}\right\}-\alpha_{3}, \quad \mu_{3, k} \geq 0, \\
v_{3, k} \geq z_{m}-\sum_{n=1}^{N}\left[A_{m^{*} n}\left(\xi_{n, k}+\varepsilon_{n, k}\right)\right], \quad v_{3, k} \geq 0, \\
\omega_{3, k} \geq \sum_{n=1}^{N}\left[A_{m^{*} n}\left(\xi_{n, k}+\varepsilon_{n, k}\right)\right]-z_{m}, \quad \omega_{3, k} \geq 0, \\
\quad m=1, \cdots, M, \quad k=1, \cdots, K, \\
\quad \sum_{m=1}^{M} d_{m} z_{m} \leq \Phi_{3}, \quad \alpha_{3}, \chi_{3} \in R^{1},
\end{gathered}
$$




$$
\begin{gathered}
A_{m}^{5} \leq z_{m} \leq A_{m}^{6}, \quad m=1,2, \cdots, M \\
\chi_{3} \geq \alpha_{3}+\frac{1}{1-\beta_{3}} \sum_{k=1}^{K} \mu_{3, k} p_{3 k}^{i}, \quad i=1, \cdots, I .
\end{gathered}
$$

The linear programming problem (WCVaR 3) minimizes the worst-case conditional value at risk-the objective of the decision-maker who takes inventory loss and opportunity loss of all products in the supply chain into consideration. In addition, $\Phi_{3}$ denotes the financial budget of the supplier for all raw material. Finally $A_{m}^{5}$ and $A_{m}^{6}$ denote the least and the most stock quantity respectively. So we can get the approximate WCVaR3 and the optimal raw material stock quantity $\mathbf{z}^{*}$ for the supplier by solving problem WCVaR 3 . Here we translate the storage quantity of raw materials into the storage quantity of products and it is feasible. According to the formulation: $z_{m}^{*}=\sum_{n=1}^{N}\left(A_{m^{*} n} * u_{n}^{*}\right), \quad m=1, \cdots, M$, we can get the inventory quantity of all products $\boldsymbol{u}^{*}$.

\subsection{Coordination Model for Supply-Production-Sale Integration}

In contrast with the above models, we combine the three linear programming (WCVaR1), (WCVaR2) and (WCVaR3) into one model based on the source demand to forecast the market risk together with corresponding weights $\left(\pi_{1}\right.$, $\pi_{2}, \pi_{3}$, where $\pi_{1}+\pi_{2}+\pi_{3}=1$ ), and all the enterprises obtain corresponding decision-making strategy about ordering, production and inventory. The market risk objective function is:

$$
\Theta=\pi_{1} \chi_{1}+\pi_{2} \chi_{2}+\pi_{3} \chi_{3} .
$$

So we build the coordination risk model as follow:

$$
\begin{aligned}
& \left(\text { WCVaR 4) } \min _{\substack{\left.\boldsymbol{x}, \alpha_{1}, \mu_{1}, v_{1}, \omega_{1}, \chi_{1}\right) \\
\left(\boldsymbol{y}, \alpha_{2}, \mu_{2}, v_{2}, \omega_{2}, \chi_{2}\right) \\
\left(\boldsymbol{z}, \alpha_{3}, \mu_{3}, \nu_{3}, \omega_{3}, \chi_{3}\right)}} \Theta=\pi_{1} \chi_{1}+\pi_{2} \chi_{2}+\pi_{3} \chi_{3}\right. \\
& \text { s.t. } \mu_{1, k} \geq \sum_{n=1}^{N}\left\{\left(a_{n}+c_{n}-r_{n}\right) v_{1, k}+\left(s_{n}-c_{n}\right) \omega_{1, k}\right\}-\alpha_{1} \text {, } \\
& \mu_{1, k} \geq 0, \quad v_{1, k} \geq x_{n}-\xi_{n, k}, \quad v_{1, k} \geq 0, \\
& \omega_{1, k} \geq \xi_{n, k}-x_{n}, \omega_{1, k} \geq 0, \\
& \sum_{n=1}^{N} c_{n} x_{n} \leq \Phi_{1}, \quad \alpha_{1}, \chi_{1} \in R^{1}, \quad A_{n}^{1} \leq x_{n} \leq A_{n}^{2}, \\
& \chi_{1} \geq \alpha_{1}+\frac{1}{1-\beta_{1}} \sum_{k=1}^{K} \mu_{1, k} p_{1 k}^{i}, \\
& \mu_{2, k} \geq \sum_{n=1}^{N}\left\{\left(b_{n}+l_{n}-g_{n}\right) v_{2, k}+\left(c_{n}-l_{n}\right) \omega_{2, k}\right\}-\alpha_{2} \text {, } \\
& \mu_{2, k} \geq 0, \quad v_{2, k} \geq y_{n}-\left(\xi_{n, k}+\theta_{n, k}\right), \quad v_{2, k} \geq 0 \text {, } \\
& \omega_{2, k} \geq\left(\xi_{n, k}+\theta_{n, k}\right)-y_{n}, \quad \omega_{2, k} \geq 0, \\
& l_{n}=v_{n}+\sum_{m=1}^{M} w_{m} A_{m^{*} n}, \quad A_{n}^{3} \leq y_{n} \leq A_{n}^{4},
\end{aligned}
$$




$$
\begin{gathered}
\sum_{n=1}^{N} l_{n} y_{n} \leq \Phi_{2}, \quad \alpha_{2}, \chi_{2} \in R^{1}, \\
\chi_{2} \geq \alpha_{2}+\frac{1}{1-\beta_{2}} \sum_{k=1}^{K} \mu_{2, k} p_{2 k}^{i}, \\
\mu_{3, k} \geq \sum_{m=1}^{M}\left\{d_{m} v_{3, k}+w_{m} \omega_{3, k}\right\}-\alpha_{3}, \mu_{3, k} \geq 0, \\
v_{3, k} \geq z_{m}-\sum_{n=1}^{N}\left[A_{m^{*} n}\left(\xi_{n, k}+\varepsilon_{n, k}\right)\right], v_{3, k} \geq 0, \\
\omega_{3, k} \geq \sum_{n=1}^{N}\left[A_{m^{*} n}\left(\xi_{n, k}+\varepsilon_{n, k}\right)\right]-z_{m}, \omega_{3, k} \geq 0, A_{m}^{5} \leq z_{m} \leq A_{m}^{6}, \\
\sum_{m=1}^{M} d_{m} z_{m} \leq \Phi_{3}, \alpha_{3}, \chi_{3} \in R^{1}, z_{m}=\sum_{n=1}^{N}\left(A_{m^{*} n} * u_{n}\right), n=1, \cdots, N, \quad k=1, \cdots, K, \\
i=1,2, \cdots, I, \quad m=1, \cdots, M, \\
\chi_{3} \geq \alpha_{3}+\frac{1}{1-\beta_{3}} \sum_{k=1}^{K} \mu_{3, k} p_{3 k}^{i}, i=1, \cdots, I, \\
\left|x_{n}-y_{n}\right| \leq \delta,\left|y_{n}-u_{n}\right| \leq \sigma .
\end{gathered}
$$

Parameters $\delta>0$ and $\sigma>0$ in the last constraint inequality of the problem (WCVaR4) are called the coordination coefficients.

For the coordination strategy (WCVaR4) above, we have made some numerical experiments to verify that it is possible to obtain the coordination strategy for the supply-production-sale integration, and at the same time we compare the influence of coordination strategy on the enterprises and the supply chain as a whole.

\section{Numerical Experiment}

(WCVaR4) is a linear programming. Here we assume that we have 4 kinds of products and 6 kinds of raw materials. The demands for these products yield certain distributions. And we also assume the two fluctuation factors that $\delta=6$ and $\sigma=9$. Let the weights of risk be $\pi_{1}=\pi_{2}=\pi_{3}=\frac{1}{3}$, and the confidence levels be $\beta_{1}=\beta_{2}=\beta_{3}=95 \%$.

For simplicity, we consider the situation that distribution function yields single distribution and mixed distributions (Experiment 1 considers the situation that the demands for all products yield normal distribution, Experiment 2 considers the situation that the demands for all products yield uniform distribution, Experiment 3 considers the situation that the demands for first two products yield normal distribution, and the demands for the other two products yield uniform distribution, Experiment 4 considers the situation that the demands for first two products yield uniform distribution, and the demands for the other two yield normal distribution. In addition, the expectation for every product is same, and so is the variance). So we have Table 1. 
Table 1. Risk decisions for different distributions.

\begin{tabular}{ccccccccc}
\hline & \multicolumn{3}{c}{ Experiment 1 } & \multicolumn{5}{c}{ Experiment 2 } \\
\hline$n$ & 1 & 2 & 3 & 4 & 1 & 2 & 3 & 4 \\
$\boldsymbol{x}^{*}$ & 22.5 & 27.0 & 29.5 & 19.4 & 24.8 & 25.8 & 29.0 & 22.0 \\
$\boldsymbol{y}^{*}$ & 28.1 & 22.4 & 29.5 & 22.5 & 29.2 & 25.2 & 30.6 & 21.3 \\
$\boldsymbol{u}^{*}$ & 30.5 & 25.3 & 30.6 & 26.4 & 27.8 & 27.1 & 32.3 & 22.0 \\
$\boldsymbol{t}^{*}$ & 28.1 & 25.3 & 29.5 & 22.5 & 27.8 & 25.8 & 30.6 & 22.0 \\
\hline & & Experiment 3 & & & Experiment 4 & \\
\hline$n^{*}$ & 1 & 2 & 3 & 4 & 1 & 2 & 3 & 4 \\
$\boldsymbol{x}^{*}$ & 29.7 & 27.6 & 29.1 & 20.8 & 25.5 & 23.8 & 31.5 & 24.6 \\
$\boldsymbol{y}^{*}$ & 31.0 & 24.1 & 30.8 & 21.5 & 27.2 & 25.2 & 32.9 & 24.9 \\
$\boldsymbol{u}^{*}$ & 28.8 & 31.1 & 32.3 & 22.1 & 29.6 & 29.3 & 33.9 & 26.2 \\
$\boldsymbol{t}^{*}$ & 28.8 & 27.6 & 30.8 & 21.5 & 27.2 & 25.2 & 32.9 & 24.9 \\
\hline
\end{tabular}

We find that no matter whatever distribution they yield, the corresponding decision variables $\boldsymbol{x}^{*}, \boldsymbol{y}^{*}$ and $\boldsymbol{u}^{*}$ hardly satisfy the equation $\boldsymbol{x}^{*}=\boldsymbol{y}^{*}=\boldsymbol{u}^{*}$. In contrast, their disparities are obvious. It shows that when enterprises in the traditional supply chain focus on their own market risk, the competitiveness of the supply chain is neglected. The differentiations of internal demands increase quickly so that the supply chain would suffer tremendous losses. So a comprehensive model considering all the risk is required. From the vertical point of view, see Experiment 1, in traditional supply chain all enterprises realize their own minimum of total market risk (\$305.5), but the operation risk (\$237.3) is neglected. In fact, the total risk is (\$542.9). The supply chain may lose its competitiveness. By means of collaboration, the integrative supply chain makes a comprehensive forecast to the source demand, and achieves a balance between the market risk and operation risk. The risk is clearly reduced, with the total risk after coordination dropping to $\$ 360.9$. The results are same in other experiments. So the integrative supply chain is effective in reducing the risk. From the horizontal point of view, let us take Experiment 1 and Experiment 2, where the demands in Experiment 1 yield normal distribution and the demands in Experiment 2 yield uniform distribution. We can see that all the risks in Experiment 1 are larger than those in Experiment 2. The risks in Experiment 3 and Experiment 4 fall within the above two. Therefore, all risks correlate with their distribution, in addition the risk when the demands yield normal distribution is larger than that when the demands yield uniform distribution.

Besides the common factors such as mutual trust and cooperation, we would like to discuss here the impacts of the factors in the above numerical experiments, and summarize the strategies as follows:

1) The enterprises should strengthen mutual trust and cooperation in the supply chain. Cooperation is the trend, and mutual trust is a bridge connecting enterprises with the market. In the processes of risk identification, risk forecast, risk 
management and risk feedback, the effect of mutual trust and cooperation shows.

2) Risk preference is an important factor for enterprises in their supply chain design. Risk preference affects the measure to and the strategy against risks. The confidence level in (WCVaR) affects the market risk of the enterprises in the supply chain. If the enterprises in the supply chain have different risk preference, especially when their risk preference are completely opposite, the market risk and total risk will increase greatly, and the supply chain will operate ineffectively. Therefore, the coherence in risk preference is important for the whole supply chain.

3) All enterprises must break all kinds of capability constraints in budget, capacity, inventory and etc. All the constraints have impact on the efficiency of the supply chain, and should be fully considered in practice. Moreover when the supply chain acts as a whole, its efficiency depends on the least efficiency part. So the supply chain should break all kinds of capacity constraints and improve themselves.

\section{Conclusions}

In this paper, a risk coordination model is proposed to cover the whole supply chain including producing, order, inventory and sales. To sum up, coordination strategy for the supply-production-sale integration dealing with the multi products can reduce the total risk and operation risk in supply chain effectively. And we are to point out that the coordination strategy is a static strategy, which provides the decision-makers with an important guidance.

We will study the risk coordination model of multiple objective loss functions to cover the whole supply chain including producing, order, inventory and sales for further research. We can even consider the situation of multi retailers.

\section{Acknowledgements}

This work is supported by the Natural Science Foundation of Zhejiang Province, Grant No. LY15G010007.

\section{References}

[1] Cohen, M.A. and Moon, S. (1991) An Integrated Plat-Loading Model with Economies of Scope. European Journal of Operational Research, 50, 266-279. https://doi.org/10.1016/0377-2217(91)90260-3

[2] Lanzenauer, C.H. and Glombik, K.P. (2002) Coordinating Supply Chain Decisions: An Optimization Model. OR Spectrum, 24, 59-78. https://doi.org/10.1007/s291-002-8200-3

[3] Nagurney, A., Cruz, J., Dong, J. and Zhang, D. (2005) Supply Chain Networks, Electronic Commerce and Supply Side and Demand Side Risk. European Journal of Operational Research, 164, 120-142. https://doi.org/10.1016/j.ejor.2003.11.007

[4] Escudero, L.F. and Schumann, E.G. (1999) A Modeling Framework for Supply Chain Management under Uncertainty. European Journal of Operational Research, 119, 14-34. https://doi.org/10.1016/S0377-2217(98)00366-X 
[5] Torabi, S.A. and Hassini, E. (2008) An Interactive Possibilistic Programming Approach for Multiple Objective Supply Chain Master Planning. Fuzzy Sets and Systems, 159, 193-214. https://doi.org/10.1016/j.fss.2007.08.010

[6] Artzner,P., Delbaen, F., Eber, J.M. and Health, D. (1999) Coherent Measures of Risk. Mathematical Finance, 9, 203-228. https://doi.org/10.1111/1467-9965.00068

[7] Rockafellar, R.T. and Uryasev, S. (2000) Optimization of Conditional Value-at-Risk. The Journal of Risk, 2, 21-41. https://doi.org/10.21314/JOR.2000.038

[8] Rockafellar, R.T. and Uryasev, U. (2002) Conditional Value-at-Risk for General Loss Distributions. Journal of Banking \& Finance, 26, 1443-1471. https://doi.org/10.1016/S0378-4266(02)00271-6

[9] Gotoh, J. and Takano, Y. (2007) Newsvendor Solutions via Conditional Value-at-Risk Minimization. European Journal Operational Research, 179, 80-96. https://doi.org/10.1016/j.ejor.2006.03.022

[10] Zhang, D. and Xu, H. (2009) Single and Multi-Period Optimal Inventory Control Model with Risk-Averse Constraints. European Journal of Operational Research, 199, 420-434. https://doi.org/10.1016/j.ejor.2008.11.047

[11] Zhou, Y., Chen, X. and Wang, Z. (2008) Optimal Ordering Quantities for Multi-Products with Stochastic Demand: Return-CVaR Model. International Journal of Production Economics, 112, 782-795.

[12] Zhu, S. and Masao, F. (2009) Worst-Case Conditional Value-at-Risk with Application to Robust Portfolio Management. Operations Research, 57, 1155-1168.

https://doi.org/10.1287/opre.1080.0684 